\title{
Transition to nonchaotic behavior in a Brownian-type motion
}

\author{
B. Kaulakys* and G. Vektaris ${ }^{\dagger}$ \\ Institute of Theoretical Physics and Astronomy, \\ A. Goštauto 12, 2600 Vilnius, Lithuania
}

\begin{abstract}
A theoretical and numerical analysis of the transition from chaotic to nonchaotic behavior in an ensemble of particles with different initial conditions which move according to Newton's equations in a bounding potential and are driven by an identical sequence of random forces (see S. Fahy and D. R. Hamann, Phys. Rev. Lett. 69, 761 (1992)) is presented. The threshold values of the parameters for transition from chaotic to nonchaotic behavior are defined on the basis of the map for distances between the particles and differences of velocity. Numerical analysis is fulfilled for one-dimensional Duffing $V(x)=x^{4}-x^{2}$ and $V(x)=x^{4}$ potentials.

PACS number(s): 05.40.+j, 05.45.+b
\end{abstract}

Recently an interesting transition from chaotic to nonchaotic behavior in randomly driven systems has been discovered [1]. When an ensemble of bounded in a fixed external potential particles with different initial conditions 
are driven by an identical sequence of random forces, the ensemble of trajectories may become identical at long times. Fahy and Hamann [1] considered a particle of mass $m$ moving according to Newton's equations in a potential $V(x)$, except that at regular time intervals $\tau$ the particle is stopped and its velocity is reset to random value chosen from a Maxwell distribution with temperature $T$. It should be stressed that for every particle of the ensemble it was given an identical, randomly chosen velocity at the start of each step of time length $\tau$. This motion is in many respects similar to Brownian motion of the particles at a temperature $T$ and is frequently used in Monte Carlo simulations [2]. However, if the time interval $\tau$ between stops is lower than a threshold value $\tau_{c}$, the final trajectories of the particles are independent on the initial conditions; all trajectories become point by point identical in time. Although the trajectory is highly erratic and random, the system is not chaotic.

The similar effect may also be observed in a more general and realistic (from the physical point of view) case, i.e., when mixing at time intervals $\tau$ some part $\alpha$ of the old velocity $\mathbf{v}^{\text {old }}$ with random velocity $\mathbf{v}^{\text {ran }}$ to get a new starting velocity $\mathbf{v}^{\text {new }}=\alpha \mathbf{v}^{\text {old }}+\mathbf{v}^{\text {ran }}[3]$. Here a threshold value $\tau_{c}$ depends on $\alpha$.

Note, that the observed effect resembles a phase transition but does not depend crucially on the dimension of the space in which the particles move. Besides its intrinsic interest, this phenomenon has some importance for Monte Carlo simulations [1] and, probably, can have influence on some processes, e.g., clustering of particles.

In the paper [1] except the discovery in the computer simulations of the 
transition from chaotic to nonchaotic behavior, the analytical estimation of the average rate of contraction of the distance between initially close together identically driven particles (for a one-dimensional potential, very short time intervals $\tau$ between stops and $\alpha=0$ ) is given. Note to the paper [4] in which the similar problem was analysed and it was found that trajectories of a noisy logistic map for various initial conditions may become point by point identical in time if the noise is sufficiently strong and independent of the initial condition. Moreover, using Langevin equation, it was shown quite generally, that nonlinear systems coupled through identical strong noises may have the same trajectory in the long time limit. However, the derivation of dependence of the threshold noise on the parameters of the system [1] is absent.

It is the purpose of this paper to present a theoretical and numerical analysis of the transition from chaotic to nonchaotic behavior in randomly driven one-dimensional systems, i.e. the dependencies of the threshold value $\tau_{c}$ on the parameters of the problem, including $\alpha$, and convergence rates of the trajectories. Generalization of the method for two- and three-dimensional systems is rather straightforward (see below). It should be noted that in the numerical calculations of the mean-square distance between particles we observe the intermittency route to chaos when $\tau \rightarrow \tau_{c}$ : mean-square distance as a function of time alternates randomly between almost clustered phases and irregular bursts of the dispersed particles. Moreover, we find a transition from chaotic to nonchaotic behavior not only for the regular time intervals $\tau$ but for the randomly changing intervals between stops, i.e. for random $\tau_{i}$ (where $i$ is the number of the step) as well. Our theoretical analysis is based 
on the mapping form of the equations of motion for the distance between the particles and the difference of the velocity of the particles.

Let us consider a particle of mass $m$ moving in a one-dimensional potential $V(x)$ which confines particles to a finite region. At a time intervals $\tau_{i}$ the particle is partially stopped and its velocity is reset to a new starting velocity $v_{i}=\alpha v_{i}^{\text {old }}+v_{i}^{\text {ran }}$. Between the stops the particle moves according to Newton's equations

$$
\frac{d x}{d t}=v, \quad \frac{d^{2} x}{d t^{2}}=-\frac{1}{m} \frac{d V}{d x} .
$$

When two particles initially at points $x_{0}$ and $x_{0}^{\prime}$ are started with velocities $v_{0}$ and $v_{0}^{\prime}$ and are driven by an identical sequence of random velocities $v_{i}^{r a n}$ at the same time intervals $\tau_{i}$, coordinates and velocities of them may accidentally draw closer to one another. The convergence of the two trajectories to the single final trajectory will depend on the evolution with a time of the small variancies of the distance $\Delta x_{i}=x_{i}^{\prime}-x_{i}$ and velocity $\Delta v_{i}=v_{i}^{\prime}-v_{i}$. Moreover, we investigate a transition from chaotic to nonchaotic behavior. Generally, such a transition may be detected from analysis of behavior of the neighboring trajectories and it is described by the Lyapunov characteristic exponents and $K S$ metric entropy of the flow of trajectories in a given region of phase space [5-9]. Further we will follow this method. Comparisons of the results of such analysis with investigations by the direct numerical simulations confirm the suitability of our theoretical approach.

From formal solutions $x=x\left(x_{i}, v_{i}, t\right)$ and $v=v\left(x_{i}, v_{i}, t\right)$ of equations (1) with initial conditions $x=x_{i}$ and $v=v_{i}$ at $t=0$ it follows an equation for 
$\Delta x(t)$ and $\Delta v(t)$ at a time moment $t$ :

$$
\left(\begin{array}{c}
\Delta x(t) \\
\Delta v(t)
\end{array}\right)=\mathbf{T}\left(\alpha ; x_{i}, v_{i}, t\right)\left(\begin{array}{c}
\Delta x_{i} \\
\Delta v_{i}
\end{array}\right)
$$

where the matrix $\mathbf{T}$ is of the form

$$
\mathbf{T}=\left(\begin{array}{ll}
T_{x x} & \alpha T_{x v} \\
T_{v x} & \alpha T_{v v}
\end{array}\right)=\left(\begin{array}{cc}
\frac{\partial x\left(x_{i}, v_{i}, t\right)}{\partial x_{i}} & \alpha \frac{\partial x\left(x_{i}, v_{i}, t\right)}{\partial v_{i}} \\
\frac{\partial v\left(x_{i}, v_{i}, t\right)}{\partial x_{i}} & \alpha \frac{\partial v\left(x_{i}, v_{i}, t\right)}{\partial v_{i}}
\end{array}\right)
$$

Note, that the similar method of investigation is used in the theory of transition to chaos in classical systems $[5,6]$. However, the motion in the form $(2)$ and (3) is represented as the non-area-preserving tangent map, while classical dynamics of the conservative systems may be represented by the area -preserving maps.

According to equations (1) and (3) matrix elements $T_{x x}$ and $T_{x v}$ satisfy the equation

$$
\frac{d^{2} T_{x}}{d t^{2}}=-\left.\frac{1}{m} \frac{d^{2} V}{d x^{2}}\right|_{x=x\left(x_{i}, v_{i}, t\right)} T_{x}
$$

while $T_{v x}=\dot{T}_{x x}, T_{v v}=\dot{T}_{x v}$ and the initial conditions at $t=0$ are:

$$
\begin{gathered}
T_{x x}\left(x_{i}, v_{i}, 0\right)=T_{v v}=1, \quad T_{x v}=T_{v x}=0 \\
\dot{T}_{x x}\left(x_{i}, v_{i}, 0\right)=\dot{T}_{v v}=0, \dot{T}_{x v}=1, \dot{T}_{v x}=-\left.\frac{1}{m} \frac{d^{2} V}{d x^{2}}\right|_{x=x_{i}} .
\end{gathered}
$$

Therefore, the dynamics of the distance between the particles $\Delta x$ and the difference of the velocity $\Delta v$ may be represented by the non-area-preserving mapping form of the equations of motion

$$
\left(\begin{array}{c}
\Delta x_{i+1} \\
\Delta v_{i+1}
\end{array}\right)=\mathbf{T}\left(\alpha ; x_{i}, v_{i}, \tau_{i}\right)\left(\begin{array}{c}
\Delta x_{i} \\
\Delta v_{i}
\end{array}\right)
$$


In general, the intervals between the resets of the velocity $\tau_{i}$ may be depending on the number of step $i$.

Further analysis of the model may be based on the general theory of the dynamics of classical systems represented as maps [5-9]. Thus, for $\alpha=0$ the Lyapunov exponent is defined as

$$
\lambda=\lim _{N \rightarrow \infty} \frac{1}{N} \sum_{i=1}^{N} \frac{1}{\tau_{i}} \ln \left|T_{x x}\left(x_{i}, v_{i}, \tau_{i}\right)\right|
$$

and may be easily evaluated numerically.

For $\alpha=1$ the map (6) is area-preserving and $\operatorname{det} \mathbf{T}\left(1 ; x_{i}, v_{i}, \tau_{i}\right)=1$, while in general $\operatorname{det} \mathbf{T}=\alpha, \operatorname{Tr} \mathbf{T}=T_{x x}+\alpha T_{v v}$ and the eigenvalues $\mu_{1,2}$ of the $\mathbf{T}$ matrix are given by equation

$$
\mu^{2}-\mu \operatorname{Tr} \mathbf{T}+\operatorname{det} \mathbf{T}=0
$$

which yields

$$
\mu_{1,2}=\frac{1}{2}\left[T_{x x}+\alpha T_{v v} \mp \sqrt{\left(T_{x x}+\alpha T_{v v}\right)^{2}-4 \alpha}\right] .
$$

So, the eigenvalues come in reciprocal pair, $\mu_{1} \mu_{2}=\alpha$. For $\left(T_{x x}+\alpha T_{v v}\right)^{2}-$ $4 \alpha<0$ the eigenvalues form a complex conjugate pair with $\left|\mu_{1}\right|=\left|\mu_{2}\right|=\sqrt{\alpha}$, otherwise the eigenvalues are real.

Among the randomly driven systems only dynamics of the harmonic oscillator with regular time intervals $\tau$ for the reset of velocity may be described exactly. In this case the $\mathbf{T}$ matrix is independent on the starting coordinate $x_{i}$ and velocity $v_{i}$, i.e.

$$
\mathbf{T}=\left(\begin{array}{cc}
\cos \omega \tau & (\alpha / \omega) \sin \omega \tau \\
-\omega \sin \omega \tau & \alpha \cos \omega \tau
\end{array}\right) .
$$


If $\alpha<1$ and $\cos \omega \tau \neq \mp 1$ modules of both eigenvalues of the $\mathbf{T}$ matrix are $\left|\mu_{1,2}\right|<1$. So there is a contraction of the distance in the phase space between the particles in each step. The transition matrix for $n$ steps $\mathbf{T}_{n}=\mathbf{T}^{n}$ may be calculated by means of diagonalisation of the matrix (10). The eigenvalues of the $\mathbf{T}^{n}$ matrix are $\mu_{1,2}^{n}$ and the Lyapunov exponents are simple $\lambda_{1,2}=\frac{1}{\tau} \ln \left|\mu_{1,2}\right|$.

Generally, the mapping $\mathbf{T}\left(\alpha ; x_{i}, v_{i}, \tau_{i}\right)$ in (6) is depending on the starting coordinates $x_{i}$ and $v_{i}$. Therefore, calculation of the mapping for $n$ steps, $\mathbf{T}_{n}=$ $\mathbf{T}\left(\alpha ; x_{i+n-1}, v_{i+n-1}, \tau_{i+n-1}\right) \cdot \mathbf{T}\left(\alpha ; x_{i+n-2}, v_{i+n-2}, \tau_{i+n-2}\right) \cdots \mathbf{T}\left(\alpha ; x_{i}, v_{i}, \tau_{i}\right)$, and of the corresponding eigenvalues are complicated problems. Further we will evaluate the averaged quantities

$$
\sigma_{1,2}=\left\langle\frac{1}{\tau_{i}} \ln \left|\mu_{1,2}\right|\right\rangle=\lim _{N \rightarrow \infty} \frac{1}{N} \sum_{i=1}^{N} \frac{1}{\tau_{i}} \ln \left|\mu_{1,2}\left(x_{i}, v_{i}, \tau_{i}\right)\right|
$$

which are analogous of the averaged Lyapunov exponent (7), characterise the rate of the exponential increase of the separation of the two initially adjacent points and are related with the $K S$ entropy of the system [5,6]. Comparisons of the threshold values $\tau_{c}$ from the direct numerical simulations with those from the criterion

$$
\sigma_{\text {largest }}=0
$$

indicate to the usefulness of the quantities (11) for analysis of transition from nonchaotic to chaotic behavior of the systems.

In Fig. 1 we show typical results of the direct numerical calculations of the mean-square distance between pairs of identically driven particles in the Duffing potential $V(x)=x^{4}-x^{2}$ vs number of steps for different time intervals $\tau$ between stops and $\alpha=0$. The averaged characteristic exponents 
as functions of time between stops, $\tau$, calculated according to Eq. (7) for Duffing and $V(x)=x^{4}$ potentials are shown in Fig. 2. Regions of $\tau$ with the positive and negative characteristic exponents correspond respectively to the chaotic and nonchaotic behaviors. Such a conclusion coincides with the conclusions from the direct numerical investigation of the systems. In Fig. 3, we show dependencies of the threshold values for transition from chaotic to nonchaotic behavior of the time $\tau_{c}$ between resets of the velocity to $v_{i}^{\text {new }}=\alpha v_{i}^{\text {old }}+v_{i}^{\text {ran }}$ on the parameter $\alpha$ calculated according to criterion $(11)-(12)$.

For the relatively short time intervals one can expand the position and velocity of the particles in the powers of time $t$. Using equations of motion (1) and relations (3) we have expansions for the matrix elements $T_{x x}$ and $T_{v v}$ :

$$
\begin{aligned}
& T_{x x}=1-\frac{1}{2 m} \frac{d^{2} V}{d x_{i}^{2}} t^{2}-\frac{v_{i}}{6 m} \frac{d^{3} V}{d x_{i}^{3}} t^{3}+\cdots \\
& T_{v v}=1-\frac{1}{2 m} \frac{d^{2} V}{d x_{i}^{2}} t^{2}-\frac{v_{i}}{3 m} \frac{d^{3} V}{d x_{i}^{3}} t^{3}+\cdots
\end{aligned}
$$

We can use expressions (13) for the analytical evaluation of the average rate of contraction of the distance between trajectories for small $\tau$. So, the result of expansion of eq. (7) according to eq. (13) in powers of $\tau$ is

$$
\lambda \tau=-\frac{1}{2 m}\left\langle\frac{d^{2} V}{d x_{i}^{2}}\right\rangle \tau^{2}+\cdots
$$

where the angle brackets denote the average with respect to $x_{i}$. After the averagement according to the Boltzmann distribution with $k_{B} T=m=1$ (instead of the average with respect to $x_{i}$ from random trajectories) we have $\lambda \tau \simeq-2.2 \tau^{2}$ and $\lambda \tau \simeq-2.0 \tau^{2}$ for the Duffing potential and $V(x)=x^{4}$ 
potential, respectively. For $\tau \leq 0.5$ these evaluations of the rate coincide with the numerical calculations in Fig. 2.

Moreover, expressions (13) may be used for the description of the systems with $\alpha \rightarrow 1$ and for short time intervals $\tau$. Introducing the notation $\alpha=$ $1-2 \varepsilon(\varepsilon \rightarrow 0)$ one can have from equations (9) and (11) the approximate expressions

$$
\begin{gathered}
\mu_{1,2} \simeq 1-\varepsilon \mp \sqrt{T_{x x}+T_{v v}-2+\varepsilon^{2}}+\cdots \\
\sigma_{1,2} \simeq \frac{1}{\tau}\left[-\varepsilon \mp\left\langle\left(T_{x x}+T_{v v}-2+\varepsilon^{2}\right)^{1 / 2}\right\rangle\right] . \\
\varepsilon=(1-\alpha) / 2 \ll 1, \quad \tau \leq \tau_{c} .
\end{gathered}
$$

The angle brackets in eq. (15) denote the average with respect to $x_{i}$ and $v_{i}$ only if $T_{x x}+T_{v v}-2+\varepsilon^{2}>0$, otherwise $\mu_{1,2}$ are complex and $\sigma_{1,2} \simeq-\varepsilon / \tau$. The threshold value $\tau_{c}$ of time interval between steps for transition from nonchaotic to chaotic behavior may be evaluated from the criterion (12).

Using the average according to the Boltzmann and Maxwell distributions (instead of the average with respect to $x_{i}$ and $v_{i}$ from random trajectories) one can investigate the behavior of the system according to equations (12)(15). This yields to the expressions of the threshold time intervals

$$
\begin{gathered}
\tau_{c} \simeq 1.44(1-\alpha) /[1+1.6(1-\alpha)], \\
\tau_{c} \simeq(1-\alpha)^{2 / 3}, \quad 0.01 \leq 1-\alpha \ll 1
\end{gathered}
$$

for the Duffing and $V(x)=x^{4}$ potentials, respectively. Here and in the numerical simulations we set $k_{B} T=m=1$. It should be noted, that the "effective temperature" of the system in such simulations is $T /\left(1-\alpha^{2}\right)$. 
In Fig. 3 the dependencies (16) and (17) are compared with those obtained from the numerical calculations according to equations (4), (9), (11) and (12). Note to the dependence of the law of the approach $\tau_{c} \rightarrow 0$ as $\alpha \rightarrow 1$ on the shape of potential.

All such numerical analysis and comparisons with the theoretical results indicate to the usefulness and fitness of the present theory for the investigation of transition from chaotic to nonchaotic behavior in randomly driven systems. Generalization of the theory to more degrees of freedom is straightforward: for two- or three-dimensional systems equations (1)-(4) and initial conditions (5) for the $\mathbf{T}$ matrix are essentially the same, only instead of one equation (4) we have the systems of two or three coupled equations and dimension of the $\mathbf{T}$ matrix is 4 or 6 , respectively. Further analysis is rather similar to that according to equations (6)-(12). The only additional complication is the diagonalisation of the multidimensional $\mathbf{T}$ matrix.

In summary, we have developed a theory for transition to nonchaotic behavior in a Brownian-type motion. Theoretical analysis based on the mapping form of equations of motion for the distance between the particles and the difference of the velocity allows to simplify the problem of investigation of transition to nonchaotic behavior and results to the expressions for the criteria of the nonchaotic motion. Theoretical results agree well with the direct numerical simulations and indicate to the possibilities of generalization of the model, e.g. to more degrees of freedom, for random values of the time intervals between the resets of the velocity and for systems driven by the random forces. 
The research described in this publication was made possible in part by Grant No. LAA000 from the International Science Foundation.

* Electronic address: kaulakys@itpa.fi.lt

† Electronic address: vektaris@itpa.fi.lt

1. S. Fahy and D. R. Hamann, Phys. Rev. Lett. 69, 761 (1992).

2. D. W. Heermann, Computer Simulation Methods in Theoretical Physics (Springer, Berlin, 1990)

3. In the paper [1] the mixing $\mathbf{v}^{\text {new }}=\alpha \mathbf{v}^{\text {old }}+\beta \mathbf{v}^{\text {ran }}$ has been introduced. However, one can without loss of generality set $\beta=1$ together with replacement of temperature $T$ by $\left(1-\alpha^{2}\right) T$.

4. A. Maritan and J. R. Banavar, Phys. Rev. Lett. 72, 1451 (1994).

5. D. Park, Classical Dynamics and Its Quantum Analogues (Springer, Berlin, 1990).

6. L. E. Reichl, The Transition to Chaos: In Conservative Classical Systems: Quantum Manifestations (Springer, Berlin, 1992).

7. A. J. Lichtenberg and M. A. Lieberman, Regular and Stochastic Motion (Springer, New York, 1983).

8. J. D. Meiss, Rev. Mod. Phys. 64, 795 (1992).

9. J. M. Ottino et al., Science 257, 754 (1992). 
Fig. 1. The mean-square distance $\left\langle\Delta x^{2}\right\rangle$ between pairs of identically driven particles in the Duffing potential $V(x)=x^{4}-x^{2}$ vs number of steps for different time intervals $\tau$ between stops. 500 independent simulations with initial conditions from the Gaussian distribution of variance $\sigma^{2}=1$ are averaged. The time $\tau$ between stops was $\tau=1.75$ for the first 300 steps and $\tau=1.3$ for the next steps.

Fig. 2. Averaged characteristic exponents (Lyapunov exponents multiplied by $\tau$ ) vs the time $\tau$ between stops according to equation (7) for (a) Duffing potential and (b) $V(x)=x^{4}$ potential.

Fig. 3. Dependencies of the threshold value for transition to chaotic behavior of the time $\tau_{c}$ between resets of the velocity to $v_{i}^{\text {new }}=\alpha v_{i}^{\text {old }}+v_{i}^{\text {ran }}$ on the parameter $\alpha$ according to criterion (11) - (12) and according to the analytical limiting expressions (16) and (17) for (a) Duffing potential and (b) $V(x)=x^{4}$ potential 